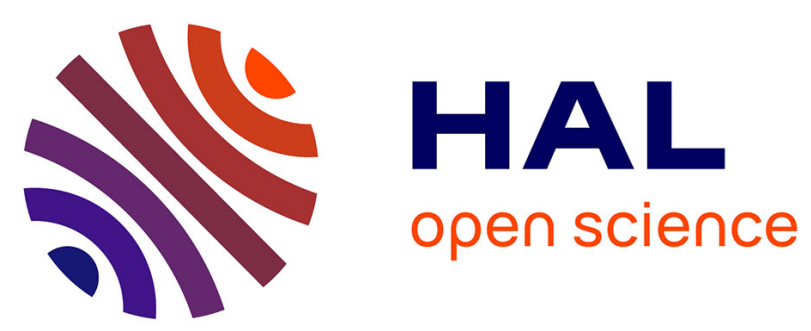

\title{
Le calcul des coûts de revient dans la sidérurgie: de la seconde guerre mondiale à la nationalisation
}

François Meyssonnier

\section{To cite this version:}

François Meyssonnier. Le calcul des coûts de revient dans la sidérurgie: de la seconde guerre mondiale à la nationalisation. 21ÈME CONGRES DE L'AFC, May 2000, France. pp.CD-Rom. halshs00587488

\section{HAL Id: halshs-00587488 \\ https://shs.hal.science/halshs-00587488}

Submitted on 20 Apr 2011

HAL is a multi-disciplinary open access archive for the deposit and dissemination of scientific research documents, whether they are published or not. The documents may come from teaching and research institutions in France or abroad, or from public or private research centers.
L'archive ouverte pluridisciplinaire HAL, est destinée au dépôt et à la diffusion de documents scientifiques de niveau recherche, publiés ou non, émanant des établissements d'enseignement et de recherche français ou étrangers, des laboratoires publics ou privés. 


\section{Le calcul des coûts de revient dans la sidérurgie : de la seconde guerre mondiale à la nationalisation}

François Meyssonnier

\section{Résumé}

Le calcul des coûts de revient dans la sidérurgie a joué un rôle plus institutionnel que managérial. Il a été organisé par la Chambre Syndicale de la Sidérurgie Française afin de négocier le niveau des prix de vente dans de bonnes conditions avec les pouvoirs publics. Par contre les systèmes d'information et d'animation autour des coûts ont été très peu développés dans les entreprises de la branche. Ce n'est qu'à la fin des années 70 qu'un contrôle de budgétaire, basé sur les coûts standards et l'analyse des écarts, a été mis en place.

Mots clés. Coûts de revient, sidérurgie, fixation des prix de vente, comportement des acteurs.

\begin{abstract}
The cost prices calculation in the iron and steel industry plays a more institutional than managerial role. It was organized by the "Chambre Syndicale de la Sidérurgie Française " in order to negotiate the price level with the authorities along favourable terms. But information and managerial systems around the costs are hardly developed in the firms of the sector. It was only at the end of the seventies that a budgetary control, based on standard costs and variance analysis, was implemented.

Key words. Cost of sales, iron and steel industry, price setting, actor's behaviour.
\end{abstract}

\footnotetext{
${ }^{1}$ Maître de Conférences à l'Université de Metz, Centre Européen de Recherche En Management des Organisations, Département G.E.A, I.U.T de Metz, Ile du Saulcy 57000 METZ, Tél. : 03873151 10, Fax : 03873151 12, Email : francois.meyssonnier@wanadoo.fr
} 
En matière de contrôle de gestion on distingue classiquement deux buts différents, la connaissance objective des coûts d'une part, l'orientation des comportements d'autre part (Bouquin, 1993). Cette analyse dichotomique est très utile conceptuellement dans l'approche des systèmes d'information et des dispositifs d'animation mis en œuvre dans les entreprises à partir des calculs de coûts. L'étude de l'évolution des pratiques managériales liées à la comptabilité de gestion montre que les choses peuvent être toutefois un peu plus complexes, notamment si on s'intéresse à la nature des acteurs dont on veut orienter le comportement.

Nous allons montrer comment dans le secteur sidérurgique, longtemps élément essentiel de notre économie, les pratiques de comptabilité de gestion ont évolué. Il nous semble que les calculs de coûts de revient ont servi entre la fin des années trente et la fin des années soixante-dix surtout à réguler collectivement le fonctionnement de la branche dans un cadre national (dimension institutionnelle prépondérante) avant de devenir à partir des années quatre-vingt un élément plus objectif d'orientation stratégique des entreprises confrontées à une concurrence accrue dans un cadre mondial (dimension économique prépondérante). La comptabilité de gestion utilisée surtout pour peser sur le comportement d'acteurs externes aux entreprises sidérurgiques (les pouvoirs publics qui contrôlent les prix de vente) ne sert que tardivement à agir sur les acteurs internes de l'entreprises (les managers qui pilotent les processus productifs).

On étudiera d'abord la place centrale du calcul du prix de revient dans le fonctionnement de la branche $(\$ 1)$ en décrivant la situation économique et institutionnelle après 1945 et en analysant la nature des rapports dans la sidérurgie entre prix de revient et prix de vente. Nous aurons l'occasion ainsi prolonger le travail de Zimnovitch (1999) qui portait sur un sujet proche : les rapports entre les pouvoirs publics et le cartel de l'aluminium autour du calcul des coûts entre 1936 et 1945.

On étudiera ensuite les méthodes et caractéristiques de la comptabilité de gestion $(\$ 2)$ en montrant l'importance du coût d'exploitation direct des produits sidérurgiques et l'évolution des

méthodes de calcul des coûts complets puis en voyant comment les informations sur les coûts sont établies et utilisées. Nous apporterons ainsi, à partir d'une approche historique et sectorielle, une contribution à la connaissance de l'évolution des comportements des entreprises en matière de comptabilité de gestion.

\section{La place centrale du calcul du prix de revient dans le fonctionnement de la branche}

\subsection{La situation économique et institutionnelle de la sidérurgie après 1945}

\subsubsection{Le développement productif et la concentration du secteur}

Après le séisme économique de l'annexion de 1870 qui l'avait privé d'une partie considérable de ses usines sidérurgiques, la France avait reconstitué, au début du $\mathrm{XX}^{\text {ème }}$ siècle, un 
ensemble productif dans l'acier suffisant pour répondre à ses besoins. Le retour des territoires annexés d'Alsace et de Lorraine en 1918 généra une surcapacité de l'outil sidérurgique qui constitua un frein objectif aux investissements nouveaux et qui limita donc pour un temps les progrès technologiques et les gains de productivité liés à la modernisation. C'est pourquoi la rationalisation et les concentrations ne s'enclencheront véritablement qu'après 1945.

Il y a trois périodes assez distinctes dans l'évolution de la branche après la seconde guerre mondiale. Jusqu'à la fin des années 60 : c'est d'abord la reconstruction et le boom lié à la guerre de Corée puis la croissance considérable des années «glorieuses » qui s'effectue avec des taux de profit très importants. Ensuite, on entre dans une phase de transition avec en 1971 un plan important de rationalisation chez Wendel-Sidélor. La production est passée, dans ces deux premières phases, de quasiment rien au lendemain immédiat de la guerre (2 millions de tonnes seulement en 1945 !) à 27 millions de tonnes en 1974 par vagues successives avec une croissance annuelle moyenne de $7 \%$ en tonnage et $8 \%$ en valeur (à francs constants). Puis la crise, qui se généralise, fait reculer, entre 1974 et 1984, la production française de 27 à 17,6 millions de tonnes et les effectifs de 158000 à 100000 personnes.

Les restructurations ont commencé au lendemain de la seconde guerre mondiale dans le secteur sidérurgique encore très éclaté. Usinor est fondé en 1948 par le rapprochement des producteurs du Nord de la France, Denain-Anzin d'une part, Nord-Est d'autre part. Symétriquement en Lorraine, en 1950, la Société des Acieries de Rombas, la Société des Acieries de Micheville et une partie des actifs des fonderies de Pont-à-Mousson fusionnent et constituent Sidélor. Toujours en Lorraine, en 1951, De Wendel SA et Les petits-fils de François De Wendel se rapprochent constituant De Wendel et Cie. Les fusions-absorptions vont devenir ensuite permanentes et permettront une concentration de l'offre autour des quatre grands des années cinquante : Usinor, De Wendel, Lorraine-Escault, Sidélor.

En 1966, pour les Nordistes, c'est la fusion d'Usinor et Lorraine-Escaut. Le poids des grandes familles (très présentes à la direction de Lorraine-Escaut) s'accroît alors dans Usinor. En Lorraine, en 1964, c'est la création de Sacilor principale filiale commune de production de Sidélor et de De Wendel. Puis la Société Mosellane de Sidérurgie, constituée en 1963 de la fusion de Knutange et UCPMI fusionne avec Sidélor et De Wendel dans Wendel-Sidélor en 1967. Un duopole est donc constitué pour une dizaine d'années : les nordistes d'un côté, les lorrains de l'autre.

En 1978, devant les extrêmes difficultés financières des entreprises, on assiste à une prise en charge par l'Etat des dettes de la sidérurgie. En 1982, c'est la nationalisation officielle puis la réunion dans le seul groupe : USINOR.

Les principaux choix stratégiques dans la branche après la guerre ont été initiés par le groupe nordiste. En 1950, Usinor lance le train à bande à Montataire permettant un accroissement de la production et une baisse des coûts suivi, en 1953, par la Sollac qui se crée et rattrape son retard. En 1963, Usinor une nouvelle fois prend l'initiative et développe la sidérurgie sur l'eau à Dunkerque. Il est copié 7 ans après, en 1970, par la Sollac qui lance Fos mais est confrontée à des difficultés et doit céder 50\% de l'opération Fos à Usinor en 1974. On peut considérer que Usinor mène le bal et que les lorrains autour des De Wendel puis de Sacilor suivent : il y a un leader 
innovateur et un suiveur imitateur. La stratégie de suiveurs des lorrains, qui dupliquent les investissements, va accroître les difficultés du secteur (Godelier, 1995).

Mais dans le fonctionnement de la métallurgie, à côté des acteurs économiques habituels et «normaux» que sont les entreprises, les organismes collectifs du secteur ont joué un rôle considérable.

\subsubsection{Le rôle essentiel de la Chambre Syndicale de la Sidérurgie Française}

La branche sidérurgique a été une des premières à se structurer du point de vue institutionnel au point de constituer le noyau dur des organisations professionnelles du patronat français. Cette organisation s'est concrétisée en tant que groupe de pression économique et politique dans le Comité des Forges et en tant qu'acteur commercial dans les comptoirs de vente des produits de la métallurgie.

Le Comité des Forges a été créé en 1864 à l'instigation d'Eugène Schneider. C'était un syndicat professionnel qui avait pour objet l'étude et la défense des intérêts économiques, industriels et commerciaux de l'industrie sidérurgique. Il fut dissout par Vichy et la Chambre Syndicale de la Sidérurgie Française (C.S.S.F) lui succéda en 1946. La Chambre Syndicale devint alors le porte-parole du patronat de la métallurgie. Financée à la tonne produite et gérant le centre de recherche collectif de la branche, l'IRSID, elle joua un rôle essentiel d'inspiration et de régulation dans la branche.

Les comptoirs, de leur côté, furent des organismes communs mis en place par les industriels pour vendre leur production. Sont mis en place par exemple le Comptoir Métallurgique de Longwy, le Comptoir d'Exportation des Fontes de Meurthe-et-Moselle, le Comptoir des Poutrelles, le Comptoir d'Exportation des Produits Métallurgiques, le Comptoir des Ressorts, le Comptoir des Essieux, etc. Ils sont unifiés en 1919 dans le Comptoir des Produits Sidérurgiques (C.P.S) qui procède à la gestion administrative des commandes jusque dans les années d'après-guerre. Le C.P.S (contrôlé par la C.S.S.F) emploie au début des années 1950 environ 750 administratifs, centralise les commandes de produits sidérurgiques, organise la répartition entre les usines, le suivi de la livraison et de la facturation des clients. Jean Constant, délégué du syndicat des industries mécaniques (regroupant donc une large part des clients des sidérurgistes) indique à ce propos: «Les utilisateurs en étaient arrivés à considérer comme normal de passer leurs commandes sans même pouvoir choisir leur fournisseur, sans pouvoir discuter ni les délais de livraison, ni les prix, ni les conditions de paiement. Pour acheter leur métal, ils n'avaient plus besoin d'acheteurs : ils pouvaient s'en remettre à une dactylo, comme s'il s'était agi d'acheter des timbres-poste. » (in, Manutention, n5, 1953).

Cette organisation corporative aurait pu changer avec la constitution de la Communauté Européenne du Charbon et de l'Acier. Celle-ci devait organiser théoriquement la libre concurrence dans un marché commun européen de l'acier. Ainsi, dans le traité instituant la C.E.C.A, l'article 65 prévoyait qu'étaient interdits tous les accords et ententes qui tendraient à fixer ou déterminer les prix, restreindre ou contrôler la production, se répartir les marchés ou les sources d'approvisionnement. Mais dans le même article, le paragraphe suivant indiquait que la 
Haute Autorité autorisait les accords de spécialisation ou d'achat et de vente en commun si cela contribuait à une amélioration dans la production ou la distribution des produits concernés !

Ce développement considérable des structures collectives dans la branche a amené certains auteurs à estimer que les patrons de la sidérurgie avaient cédé une part très importante, voire essentielle, de leurs responsabilités à l'organisation professionnelle (Padioleau, 1981). Le rôle longtemps stratégique de l'acier dans le développement de l'économie explique par ailleurs l'intérêt particulier que l'Etat accordait à la branche et les conséquences de ceci sur les modes de régulation des marchés.

\subsection{La nature des rapports entre prix de revient et prix de vente dans la sidérurgie}

\subsubsection{Le contrôle des prix et la connaissance des coûts}

Le contrôle des prix est instauré en 1937. Les prix restent bloqués jusqu'en 1953 mais les entreprises bénéficient de subventions compensatoires. Les prix de l'acier sont ensuite toujours plus ou moins contrôlés jusqu'aux années 80. Cette situation, qui fait que dans la métallurgie les prix ne peuvent évoluer qu'avec l'accord de la puissance publique, implique des rapports permanents et étroits entre l'Etat (par ses deux directions concernées : celle de la sidérurgie au Ministère de l'Industrie et celle du contrôle des prix au Ministère des Finances) et la Chambre Syndicale de la Sidérurgie Française (qui dispose d'un appareil administratif conséquent). Les entreprises sont totalement en retrait, déléguant leur pouvoir de négociation à la Chambre.

Les relations entre l'Etat et la sidérurgie sont particulièrement développées comme le montre Mioche (1992). Ainsi beaucoup des ingénieurs des mines, après une période de service comme fonctionnaires, deviennent des cadres des entreprises ou des institutions du secteur. Par exemple, Albert Bureau, le très puissant Directeur de la Sidérurgie au Ministère de l'Industrie dans l'immédiat après-guerre, fut ensuite salarié de la C.S.S.F et conseiller spécial du président Ferry.

Pour que l'Etat puisse fixer les prix de vente maximum il est nécessaire qu'il connaisse les coûts constatés réellement dans les usines du secteur. Effectuer des comparaisons avec les prix de marché dans les autres pays apporte relativement peu dans un marché mondial assez fragmenté et avec des situations très différentes des outils de production nationaux. La puissance publique qui souhaite développer la capacité de production nationale doit partir de l'existant, ou du moins de ce qu'elle en sait. Or son seul interlocuteur, son fournisseur exclusif d'informations en matière de coûts de production, c'est la Chambre Syndicale. Jacques Ferry, qui fut longtemps le président de la C.S.S.F, reconnaît ainsi : «L'Administration a plusieurs fois cherché à connaître nos prix de revient. Elle a même voulu les obtenir directement des entreprises. Nous avons toujours refusé. ».

Le secret sur les calculs de coût de revient était jugé essentiel dans la branche. Cette information ne devait surtout pas parvenir directement à l'environnement institutionnel. Le détail des modalités du calcul et les chiffres de référence devait rester confidentiel comme le montre, parmi beaucoup d'autres, la note de la C.S.S.F du 13 août 1948 qui indique qu'il faut prendre des dispositions «pour qu'il n'y ait aucune fausse manæuvre de la part des usines et qu'aucune 
d'entre elles n'accepte de se laisser directement questionner par la Direction de la Sidérurgie ou l'Ingénieur des Mines » (62 AS 92). Ce comportement était aussi très fort en interne au niveau des directions d'usine et d'entreprise : la connaissance des coûts de revient était la prérogative exclusive du top-management comme en attestent tous les témoignages recueillis sur cette période (Mioche 1992, Sallot 1993, Godelier 1995). Les ingénieurs du rang et les cadres intermédiaires jusqu'à un niveau assez élevé n'y avaient pas accès (à l'inverse de ce que l'on essaie de développer actuellement dans l'industrie).

L'Etat a bien tenté, à une occasion, de se procurer l'information à la source afin d'éviter de dépendre exclusivement du bon vouloir de la Chambre Syndicale. C'est en 1947 que l'administration décida d'effectuer une enquête sur le terrain pour avoir une idée précise des coûts de revient de la branche indépendamment de la Chambre Syndicale.

Si on avait envisagé dans un premier temps à la direction de la sidérurgie au Ministère de l'Industrie, fin 1946, de fixer les prix de façon indépendante des coûts de revient afin d'orienter par l'aval la production sidérurgique en décourageant par exemple la fabrication de certains produits lourds (poutrelles, rails, etc.) et en encourageant la fabrication d'autres produits (tubes, ronds béton, fil machine, etc.), cette proposition fut vite abandonnée sous la pression de la profession. On décida donc de fixer les prix de vente à partir des coûts de revient. On fit une estimation à partir du coût de revient moyen (en prenant la moyenne des coûts d'une usine récente, d'une usine intermédiaire et d'une usine vieillotte) et non pas du coût de revient des usines les plus performantes. En raison de l'hétérogénéité du secteur et de sa faible rationalisation, cela permit à de nombreux sidérurgistes de dégager des profits considérables.

De plus l'enquête "n'a donné aucun renseignement sérieux sur les variations du prix de revient en fonction du taux de marche » indique le directeur de la Sidérurgie au Ministère de l'Industrie dans une lettre du 6 septembre 1948 (62 AS 92). Ceci est confirmé plus tard du côté de la C.S.S.F par Ferry qui se félicite que «on ne peut rien tirer de l'examen des chiffres sur l'évolution des prix de revient par rapport au taux de marche» lors de la réunion prix du 14 février 1951(62 AS 93). La diminution des coûts de revient rendue possible par l'accroissement de la production et la ventilation des frais fixes sur un plus grand nombre de produits est donc ignorée !

Dans ces conditions la Chambre Syndicale se satisfera finalement de cette enquête publique dont les résultats ne remettront pas directement en cause le niveau des prix et l'organisation de la profession. Au début des années 1950, les prix des produits sidérurgiques français seront supérieurs d'environ 30\% aux prix des produits sidérurgiques allemands (Kipping, 1994)!

Par la suite tous les calculs de coûts se feront sur la base des méthodes prônées par la C.S.S.F et des résultats qui en sont issus. Les chiffres perdent de plus en plus de leur pertinence au fil des réajustements forfaitaires successifs. Par ailleurs on n'hésite pas à les orienter quelque peu. Ainsi par exemple lors de l'enquête menée par le sénateur Armengaud en 1954 sur la sidérurgie, un document interne de la C.S.S.F indique comment on a manipulé les données et les méthodes de calcul transmis au sénateur afin d'aboutir au résultat souhaité. : "Il est inutile de rappeler le peu de sérieux qu'il faut attribuer à ces documents. (...) Parmi l'assortiment de coefficients que l'on pouvait recueillir dans les statistiques allemandes, on a choisi ceux qui 
conduisaient au résultat souhaité sur la fonte Thomas. » (note de Poulain à Ferry du 13 février 1954).

Il est alors logique que la conclusion du rapport soit que les entreprises sidérurgiques sont bien gérées et performantes mais que les prix de vente sont plus élevés qu'en Allemagne en raison uniquement de causes externes au secteur: le coût de l'énergie, les frais de transport, la fiscalité, les comportements antiéconomiques de l'Etat, etc. !

\subsubsection{L'organisation du calcul des coûts de revient par la Chambre Syndicale}

La relation qui doit exister entre coût de revient et prix de vente est une question qui suscite depuis toujours des controverses dans l'industrie. Dés 1933, lors des débats du groupe de travail sur les prix de revient de la CEGOS relatés par Zimnovitch (1997), on constate que certains comme Mr Landauer (PDG des établissements Landauer) insistent sur le fait que le prix de vente est fixé par la concurrence sur les marchés et que le calcul du prix de revient ne se justifie que pour faire progresser la gestion de l'entreprise ( le prix de vente conditionne le prix de revient»). Ce point de vue est fortement soutenu par Mr Lapiquonne représentant la société Renault mais provoque une réaction indignée de $\mathrm{Mr}$ Ollivier, président des syndicats représentatifs de la métallurgie qui dans un courrier d'octobre 1933 indique : «Nous tenons à nous élever contre cette manière de voir. (...) L'opinion générale des industriels de notre groupe est à l'opposé : on ne sortira de la crise actuelle qu'en partant de la considération du plus juste prix de revient et en ajoutant à ce plus juste prix de revient un pourcentage de bénéfices très raisonnables. ».

Il y eut donc un débat entre deux franges du patronat sur cette question centrale de la place du coût de revient. Les sidérurgistes qui font du «business to business » et qui se sont dotés d'une structure collective forte veulent profiter de leur pouvoir de négociation pour fixer les prix de vente à partir de leurs coûts de revient. Les représentants de l'industrie des biens de consommation sont plus sensibles à la logique des marchés et ouverts à la libre concurrence. Il y a aussi probablement dans ces positions antagonistes des dimensions politiques non négligeables et des intérêts économiques directement contradictoires (les uns sont clients des autres).

En tous cas, dans la sidérurgie, la première orientation s'imposa. Le calcul des coûts dans la profession fut une démarche collective bien éloignée de la norme classique qui fait de la comptabilité industrielle un domaine strictement interne à l'entreprise. Le président de la Chambre Syndicale, Jacques Ferry, indique la nature du système mis en place : "Il n'y avait pas à ma connaissance d'organisation patronale à qui ses adhérents communiquassent comme chez nous, régulièrement, leurs prix de revient. Cette communication était normalisée, sur des bases et selon des critères qui avaient été établis en commun avec les entreprises et qui étaient devenus la règle. Nul ne pouvait s'y soustraire. La Chambre Syndicale procédait régulièrement à des enquêtes ou des vérifications dont les conclusions lui permettaient de redresser certaines erreurs ou omissions. » (Mioche 1993). Il justifie cette pratique en affirmant qu'il fallait être en mesure, à tous moments, de motiver les demandes à la Direction des Prix et être capable de ventiler les hausses accordées entre les différentes qualités d'acier. 
On est là dans la filiation des conceptions d'Auguste Detoeuf dans son texte célèbre sur le problème des prix de revient (illustré par l'exemple des choux et des carottes) où il indiquait à propos des différentes méthodes de calcul des coûts : «Nous nous nuisons l'un à l'autre parce que nos méthodes de calcul sont différentes, tandis que nous ne nous serions pas nui si nous avions adopté la même. » (Detoeuf 1937). La sidérurgie a adopté, dans le face à face avec l'Etat, le point de vue d'un certain nombre de dirigeants de l'industrie des années 30 proche de la mouvance techno-corporatiste.

Le système mis en place correspondait aussi très directement aux recommandations pratiques que Rimailho développait avant-guerre : "Pour organiser la profession, il faut commencer par doter sa chambre syndicale de l'outil d'investigation qu'est une méthode générale d'établissement des prix de revient. » (Rimailho 1939).

Il ne fut pas installé sans débats comme le reconnaît Jacques Ferry : «En fait, il a fallu plusieurs années pour obtenir de nos adhérents un consentement général et sans réticences au système. Il y a eu quelques accrocs, quelques velléités de refus. Mais finalement tout est rentré dans l'ordre, l'unité de la profession apparaissant bien dans ce domaine, compte tenu des contraintes qui nous étaient imposées par les pouvoirs publics, comme l'unique rempart contre le dirigisme administratif sur les entreprises. » (Mioche 1993).

Il y eut ensuite quelques utilisations plus directement managériales dans la profession permettant une forme de benchmarking indique Jacques Ferry (dans un plaidoyer a posteriori) : «Pendant longtemps, chaque entreprise ne connaissait que le prix moyen professionnel, par grande catégorie de produits, tel que le lui communiquait nos services: elle pouvait ainsi se situer par rapport à la moyenne. Par la suite nos adhérents ont accepté que cette communication fasse état du prix de revient le plus bas et le plus élevé, c'est à dire que chacun savait quelle place il occupait par rapport aux deux branches de la fourchette. Nous avons perfectionné la méthode et, avec l'accord des intéressés, nous leur donnions connaissance, toujours avec l'indication du maximum et du minimum, des principales composantes de ces prix de revient moyens professionnels, à savoir, par exemple, la moyenne des quantités et des prix du coke enfourné ou du minerai, des salaires, etc. Chaque entreprise pouvait ainsi repérer ses forces et ses faiblesses et savoir dans quel sens il convenait de porter ses efforts pour améliorer sa compétitivité.» (Mioche 1993).

\subsubsection{Comment concilier des coûts de revient élevés et des comptes d'exploitations florissants}

On est confronté à un paradoxe: les prix de vente acceptés par l'Etat sont à peine supérieurs aux coûts de revient « officiels » de la Chambre Syndicale et pourtant la rentabilité du secteur est très forte pendant toute la période. Les explications sont à rechercher au niveau des amortissements et des prix de cession interne.

\footnotetext{
${ }^{2}$ Dans la pratique cet usage managérial des chiffres collectés et traités par la Chambre Syndicale fut probablement moins développé que ne l'affirme Ferry (comme nous le verrons clairement plus loin).
} 
A partir de 1942 et jusqu'en 1952 un système d'Amortissement Technique a été mis en place. Il joua un rôle essentiel au moment où les fondements du système de fixation des prix de vente sur les marchés (déterminés par les pouvoirs publics) à partir des coûts de revient (calculés sur la base des informations de la C.S.S.F) étaient posés. Le système était le suivant: pour permettre au secteur sidérurgique de maintenir et moderniser l'outil de production malgré le blocage des prix on prélève sur les ventes de produits sidérurgiques une marge (à la tonne et différente selon les produits) qui vient alimenter un fonds qui permettra le renouvellement des équipements actuels (sans financer une extension). Ces montants, appelés Amortissement Technique (A.T.), calculés sur la base des prix contemporains d'installations neuves, sont largement supérieurs aux amortissements fiscaux, calculés eux sur la base du coût historique d'installations très anciennes. Pendant quelques années ceci a été très favorable aux entreprises (ainsi sur les trois années 1948-1949-1950, l'A.T. a dépassé l'amortissement fiscal de 35\% soit presque 10 milliards de $\mathrm{F}$ de l'époque).

L'A.T était intégré aux calculs des coûts de revient effectués par la C.S.S.F (majorant donc leur valeur de façon «artificielle »). Les fonds collectés étaient reversés aux entreprises et devaient servir à financer les travaux neufs assurant la modernisation de l'outil de production. Si ces travaux étaient réellement engagés, ils généraient ensuite des amortissements fiscaux plus élevés qui réduisirent progressivement l'intérêt de l'A.T par rapport à l'amortissement classique.

En 1947 le montant de l'amortissement technique était fixé dans les arrêtés de prix. Ce n'est plus le cas en 1951 : les arrêtés indiquent seulement le montant des versements effectués dans un compte spécial ouvert dans les écritures du CPS au crédit de chacune des usines productrices pour couvrir les dépenses dûment justifiées de renouvellement des installations, du matériel sidérurgique et de constructions de logement.

Une note de la C.S.S.F adressée en 1951 à toutes les usines de la sidérurgie par J. Ferry, représentative de nombreuses notes du même type, indique comment faire au sujet de la prise en compte de l'amortissement technique dans le calcul des coûts de revient (référence 42/18, Archives d'USINOR). Considérant que les montants de l'amortissement technique en 1947 (pour les laminés Thomas : $1690 \mathrm{~F}$ et pour les laminés Martin : $1440 \mathrm{~F}$ ) avaient été calculés en prenant en compte les salaires (pour 42\%), les produits sidérurgiques (pour 43\%) et les produits réfractaires et huiles (pour 15\%), la note comptabilise l'augmentation des prix sur chacun de ces éléments depuis 1947 et en déduit une hausse moyenne à appliquer sur l'amortissement technique de 190\% (ce qui donne une nouvelle valeur pour les laminés Thomas de $4900 \mathrm{~F}$ et pour les laminés Martin de 4180 F).

A partir de cela, la note donne un tableau détaillé des montants d'amortissement technique à appliquer pour chaque variété de produit (rails, laminés marchands, bandes, tôles, ronds, etc.) suivant le procédé de production utilisé. Elle rappelle également: «En définitive, les prix de revient totaux comprennent, en outre des prix de revient au stade usine : les frais commerciaux et de siège social, les montants de l'amortissement technique indiqués dans le tableau, les subventions versées à la tonne pour la formation professionnelle et le financement de la recherche, les cotisations au CPS. »

Par ailleurs, dans les coûts de revient usine, on prend en compte les matières premières et composants qui sont généralement livrées à un prix de cession interne par d'autres entités relevant des groupes sidérurgiques. Ceux-ci sont en effet fortement intégrés : ils réunissent sur des sites communs les différentes étapes de la filière dans une intégration verticale très poussée 
(en Lorraine cela va des mines de fer aux produits finis achevés comme à Longwy ou dans la vallée de la Fensch). Les entreprises ont donc la possibilité de faire émerger le profit là où cela leur semble le plus acceptable en manipulant les prix de transfert : ainsi le minerai est enregistré au «prix commercial » (et non pas au coût réel de production) et les ferrailles d'auto-production incluent le coût de transports fictifs !

On comprend dans ses conditions que certains utilisateurs en aval protestent en considérant que le pouvoir de négociation des métallurgistes est trop fort et qu'il y a abus de position dominante. Ainsi on relate que Pierre Lefaucheux, dirigeant de Renault, affirmait publiquement à la fin des années 40 que "les hauts fourneaux pissaient de l'or» (note du 18 mars 1948, IND 22 298). Jean Sallot (1993) démontre qu'en 1948 les profits de la métallurgie ont cru de $160 \%$. Il cite également une enquête de la direction des prix en 1951 qui constate que les sociétés sidérurgiques dégagent des profits conséquents qui, après constitution des réserves, leur permettent de distribuer des dividendes importants à leurs actionnaires, alors que, d'après les experts, les prix de vente sont inférieurs de $6 \%$ aux coûts de revient. Ceci en raison de manipulation des prix de cession et du fait que «il suffit que les amortissements fiscaux soient inférieurs aux amortissements techniques pour que des bénéfices puissent être légalement dégagés ».

Il apparaît que chaque fois que les pouvoirs publics fixent les prix de vente à partir de coûts de revient établis par les industriels sans s'être dotés des moyens d'investigation, de contrôle et d'analyse suffisants (le cas de la sidérurgie rejoignant le cas de l'aluminium étudié par Zimnovitch, 1999), ils aboutissent à des résultats insatisfaisants ${ }^{3}$.

Ceci nous conduit donc logiquement à nous intéresser de façon plus approfondie aux techniques de calcul et d'analyse des coûts mises en œuvre dans la sidérurgie.

\section{Méthodes et usages de la comptabilité de gestion}

\subsection{Modalités des calculs de coûts}

\subsubsection{Le rôle central du coût d'exploitation direct}

Zimnovitch (1997) distingue plusieurs périodes en matière de calcul des coûts de revient en France. Pendant la période 1880-1930, on élabore des prix de revient comptable polarisés sur la précision de l'affectation des matières premières et de la main-d'œuvre dans le coût de revient (quête de légitimité de la profession comptable). Ensuite, de 1930 à 1950, on met au point un prix de revient uniforme avec une ventilation fine des charges indirectes aux différentes étapes de la production (idéologie technocratique et corporatiste des ingénieurs). Enfin, à partir de 19501960, les coûts standards se généralisent, sous l'influence des missions de productivité, permettant la confrontation du réel et du prévu et l'analyse des écarts. Il en est de même pour le

\footnotetext{
${ }^{3}$ On ne peut ici s'empêcher de penser à certaines situations actuelles où des problèmes analogues se posent de façon cruciale...par exemple dans le cas du marché de l'eau et des rapports entre collectivités locales et grandes entreprises du secteur... ou dans le cas de la fixation par les autorités régulatrices des tarifs de facturation de l'usage des infrastructures de télécommunications par les différents opérateurs.
} 
direct costing qui, par la prise en compte des coûts partiels, permet les ajustements aux fluctuations d'activités, boom ou crises.

Dans la sidérurgie on semble avoir suivi la même évolution avec toutefois un retard certain dans le passage à l'étape des coûts standards et des analyses d'écarts. Le prix de revient est en général calculé ex-post, hors coût réel des amortissements, sans imputation rationnelle des charges fixes, et sert essentiellement à négocier avec les pouvoirs publics.

Depuis toujours dans cette branche (comme en témoigne par exemple le cas de Decazeville en 1830 étudié par Nikitin (1996)), on assimile le prix de revient de référence à un prix d'exploitation regroupant les charges directes de production : matières premières et autres matières, main-d'œuvre de production et frais divers liés comme les frais de transport. Les dotations pour le gros entretien n'interviennent pas dans ce coût. Les amortissements ne sont pas pris en compte dans le coût usine. Ils interviennent parfois, comme dans les calculs de Chambre Syndicale, mais après que le total usine ait été fait. Et nous avons montré qu'à ce moment, ils sont plus des conventions (l'Amortissement Technique par exemple est estimé forfaitairement à la tonne de produit pendant 10 ans dans l'après-guerre) que des montants calculés précisément sur la base des investissements réellement effectués dans l'usine.

Les calculs de coûts restent ainsi assez frustes et pas seulement en France. Bouquin (1993) relève qu'actuellement encore diverses enquêtes menées aux Etats-Unis montrent que dans certaines branches, dont la sidérurgie, l'intégration aux stocks de charges fixes comme l'amortissement est peu pratiqué.

Nikitin (1992) a démontré que les premiers développements de la comptabilité analytique étaient lié à la révolution industrielle. Les nouveautés dans les méthodes du contrôle de gestion qu'analyse Zimnovitch et notamment les budgets et les analyses d'écarts, qui se sont développés en France à partir des années 1950, sont essentiellement le fait d'entreprises liées à la deuxième industrialisation (comme l'automobile). Dans la sidérurgie le contrôle budgétaire n'apparaîtra qu'au début des années quatre-vingt, avec trente ans de retard ! Ceci illustre peut-être un certain fixisme dans les modes de régulation des entreprises, comme le suppose tout le courant de pensée qui se réclame de l'écologie des populations d'organisations (depuis Hannan et Freeman, 1977). Une raison probable est donnée par un cadre d'USINOR : "Il faut bien voir que les clients font alors la queue au service commercial de Valenciennes. Celui-ci augmente les prix dés qu'il le peut et engrange des bénéfices tels que personne ne les avait prévus. (...) Si vous vivez durant 30 ans dans de telles conditions, vous avez du monde une vision qui n'est pas du tout celle du couteau entre les dents d'un monde compétitif tel qu'il existe actuellement. » (cité par Godelier, 1995). Dans ces conditions, l'utilisation des coûts standards et de l'analyse d'écarts ne semble pas indispensable !

On doit aussi noter que, dans le pilotage des centres de production, les coûts maîtrisables sont essentiellement ceux directement liés à l'exploitation. D'un certain point de vue, sauf aux moments exceptionnels de crises majeures et de choix stratégiques déterminants, les équipements sont donnés et leur coût ne change pas grand chose aux choix courants de gestion. Il faut souligner, à ce propos, que, surtout en Lorraine, on a hérité de sites de production d'origines diverses au gré des fusions avec une interpénétration inévitable d'équipements vétustes et 
d'équipements modernisés sur un même lieu. Ceci explique peut-être la focalisation logique sur le nombre très restreint des dépenses maîtrisables.

\subsubsection{Un changement dans le mode d'imputation des frais généraux}

Les pratiques de calcul des coûts complets ont subi des évolutions intéressantes pendant la période. Le 21 décembre 1949, la Chambre Syndicale établit une note sur les coûts de revient (référence 42/18, Archives d'USINOR) qui reprend et approfondit une note du 21 décembre 1943. Les indications qu'elle donne aux entreprises portent sur certains points jugés très importants.

Elle conseille de limiter le plus possible le montant des charges considérées comme indirectes dans les calculs : "Toutes les dépenses qui peuvent être imputées directement à des prix de revient particuliers doivent l'être : c'est un principe qu'il est essentiel de ne jamais perdre de vue. En particulier les frais communs, depuis les services auxiliaires jusqu'à la direction incluse, ne doivent comprendre que les dépenses qui ne peuvent être divisées que par l'intervention d'une règle d'imputation à caractère théorique et toujours un peu artificiel. ».

Pour l'affectation des frais communs à plusieurs fabrications et des frais indirects d'usine, la Chambre Syndicale prône l'utilisation de la Main d'CEuvre Directe de fabrication et d'entretien comme clé de répartition. Pour les frais commerciaux, elle propose de les abonner sur les coûts de production usine "avec des coefficients variables si l'entreprise le juge utile pour se rapprocher des charges effectives en fonction de la nature des produits vendus. ».

Pour les «frais généraux sociaux», la Chambre Syndicale n'a pas de position tranchée, elle laisse le choix aux entreprises entre trois clés de répartition : la MOD ou une partie MOD, une partie Coût de Production ou le Chiffre d'Affaires.

La méthode de ventilation des charges indirectes change ensuite dans les années 1960. Nous avons retrouvé dans les Archives d'USINOR (référence 42/18) des notes du 7 février et du 22 mars 1963 fixant les nouvelles normes en matière de comptabilité industrielle chez De Wendel ${ }^{\dagger}$.

Ces notes organisent le passage de la ventilation sur la base de la MOD ou de la tonne à une ventilation proportionnelle à la Valeur Ajoutée : "Il n'est plus concevable d'utiliser les bases de répartition des frais généraux précédemment admises, c'est à dire le montant des salaires pour les frais $A, B, C, D$ et la tonne lingot pour ce qui est des frais du siège, ni de véhiculer, en comptabilité, ces frais à travers les prix de revient et les comptes de stocks. » (note relative à la comptabilité industrielle du 7 février 1963 chez De Wendel). La raison en est donnée : «La base de répartition antérieure des salaires qui s'approchait de la réalité tant que les salaires formaient une part prépondérante des frais de transformation, s'en écarte de plus en plus, au fur et à mesure de la généralisation de la mécanisation et de l'automation ».Nous avons là des remarques qui ressemblent beaucoup à certains des arguments développés un quart de siècle plus tard par les partisans de la comptabilité par activités !

\footnotetext{
${ }^{4}$ On retrouve les documents des métallurgistes de l'Est aux Archives d'USINOR en raison de la fusion en un seul groupe de toute la sidérurgie française dans les années quatre-vingts.
} 
Il est donc décidé d'utiliser la valeur ajoutée comme clé de répartition et de n'affecter les frais généraux qu'au stade ultime «revient-vente». La note indique la nouvelle façon de procéder: "La masse des frais généraux rapportée à la masse des valeurs ajoutées donne un pourcentage de frais généraux à appliquer au franc de valeur ajoutée. » Pour permettre de sortir les coûts sans prendre de retard, il est indiqué qu'il faut recourir à des «prix d'ordre » c'est à dire de déterminer par avance pour une période donnée les montants des valeurs ajoutées au différents stades et le montant des frais généraux à ventiler. Ces montants seront budgétisés pour une période donnée et révisés périodiquement pour prendre en compte le taux de marche des installations et le montant réel des frais généraux.

La note de février 1963 règle aussi quelques points techniques qui semblent avoir été traités de façon parfois étonnante. L'évaluation des stocks est précisée : «Il ne faudra plus réintroduire dans les stocks, comme par le passé, les frais dits commerciaux et par mesure d'unification l'amortissement technique sur les produits finis. (...) Par contre, à partir de l'exercice 1963, il sera nécessaire pour se conformer aux prescriptions fiscales sur l'évaluation des stocks, de réintégrer (en plus de l'amortissement fiscal) les frais généraux.» Certaines dérogations aux règles de ventilation sont reconduites (sans qu'il soit jugé nécessaire de justifier ce choix): "Certaines fabrication: Briqueterie, Cartoucherie, produits du moulage pour la propre consommation et les activités annexes, etc. ne prendront que des charges $A, B, C, D$ (ou $U$ ) afin de conserver le principe ancien selon lequel elles ne supportent pas de frais généraux du Siège, ni de charges financières, ni d'amortissements. ».

Nous n'avons pas pu vérifier si le même changement avait lieu simultanément dans les autres entreprises du secteur, mais nous le supposons ${ }^{\mathrm{b}}$.

\subsection{Les pratiques évoluent}

\subsubsection{L’analyse comparée des coûts}

En général dans la sidérurgie, les prix de revient sont envoyés le 30 du mois suivant sous une forme résumée et 15 jours après (soit à 45 jours) sous une forme détaillée. Il n'y a pas de réel contrôle budgétaire avec analyse d'écarts et gestion par exceptions. Les directions d'entreprise se font communiquer les prix de revient chaque mois et en discutent lors de leurs réunions de coordination mensuelles.

Les directeurs d'usine doivent justifier les évolutions de leurs coûts d'exploitation. Ceci fait partie, avec les résultats commerciaux du mois précédent et les indications sur la marche des usines, des indicateurs pris en compte systématiquement. Mais on voit que ces éléments ne sont pas raccordés ni chiffrés de façon synthétique : on analyse séparément les ventes, la production et les charges d'exploitation. Les coûts d'exploitation « usine » ne permettent de voir que les dérives éventuelles sur certains coûts d'approvisionnement, une partie des charges salariales et certains frais généraux. On n'a pas d'analyse globale, articulée et exhaustive des écarts sur l'ensemble des composantes des charges et des produits.

\footnotetext{
${ }^{5}$ Sauf dans certaines entreprises comme la S.M.S. ainsi que nous le verrons plus loin.
} 
Les échanges d'information entre les cadres dirigeants des groupes sidérurgiques sur les coûts existent toutefois. Ainsi nous avons pu retrouver un courrier, en date du 13 décembre 1965, où le chef de la comptabilité d'Usinor (à Valenciennes) communique le détail des calculs de coûts des hauts-fournaux de Dunkerque au directeur de la comptabilité de De Wendel à Hayange (référence 10/45, Archives d'USINOR). Il semble que ceci arrivait de temps en temps.

Par ailleurs, contrairement à ce que déclarait Jacques Ferry, les coûts complets transmis à la C.S.S.F n'étaient pas très fiables et que les pratiques de benchmarking se faisaient plutôt (en tous cas, dans la dernière phase du processus de concentration de la sidérurgie) d'entreprise à entreprise.

Une note interne chez De Wendel, datée du 19 juillet 1967, de la DGMU au service d'analyse économique des prix de revient, en témoigne: «Les Sociétés Sidérurgiques communiquent périodiquement leurs prix de revient à la Chambre Syndicale de la Sidérurgie. Le calcul de ceux-ci est censé se faire sur la base d'une règle commune, mais nous savons par expérience qu'il n'en est rien et que les tableaux de comparaison des prix de revient professionnels de la fonte et des aciers Thomas et Martin diffusés périodiquement n'ont pas grande valeur. D'ailleurs, chaque fois qu'une comparaison sérieuse de prix de revient doit être faite, elle se déroule en dehors de la Chambre Syndicale (comparaison SIDELOR-De Wendel, USINOR-De Wendel, DILLING SIDELOR-De Wendel). Il ne nous semble pas qu'il y ait beaucoup d'inconvénients à nous écarter une fois de plus de la doctrine syndicale comme nous nous en écartons déjà en ce qui concerne l'énergie électrique et les frais généraux... »

Mêmes si les méthodes de calcul des coûts peuvent être différentes et si les entreprises sont tentées de reprendre, petit à petit, plus d'autonomie vis à vis de la Chambre Syndicale (d'autant plus que la concentration réduit le nombre des entreprises significatives dans le secteur à quatre puis deux) ceci ne change pas totalement la donne. Ainsi le 31 janvier 1968, c'est encore sous l'égide de la Chambre Syndicale que se tient une réunion de travail pour fixer les objectifs de prix de l'acier pour l'horizon 1972.

La réunion regroupe deux représentants d'Usinor, deux de la SOLLAC, un de De Wendel et deux de la Chambre Syndicale. La comparaison des coûts de l'acier met en évidence un avantage de $17 \mathrm{~F}$ à la tonne pour les nordistes (187 F/tonne) par rapport aux lorrains (204 F/tonne). Une grande partie de l'écart semble venir des frais généraux : ceux d'Usinor étant nettement inférieurs. Les participants attribuent cela à l'existence d'une structure administrative unifiée dans le Nord alors que ce n'est pas le cas en Lorraine (écart donc réel dans les coûts) mais aussi à des différences dans le traitement de ces frais d'un point de vue comptable (écart dû aux modes de ventilation). Ils se penchent ensuite sur les coûts usine et mettent en évidence des imprécisions : "Le détail des prix de revient fonte dans la région de Thionville fait apparaître un prix de main d'œuvre horaire supérieur pour la société S.M.S. par rapport aux sociétés De Wendel et Sidélor. La S.M.S. enchaînant ses frais généraux en les véhiculant par les salaires, Monsieur Charrier est chargé de s'assurer auprès d'Hagondange que les frais généraux ont bien été expurgés et qu'ils ne sont pas à l'origine de la disparité des prix horaires. » On voit que, dans certaines entreprises, on a gardé les méthodes anciennes de ventilation sur la base de la MOD et qu'on n'est pas certain que coût du facteur (salaire) et coût de l'unité d'œuyre (montant des charges indirectes affectées sur la base de ce critère) n'aient pas été additionnés !

\footnotetext{
${ }^{6}$ Le recours à des "taux chargés" mélant charges directes et indirectes est assez courant mais n'est pas une bonne chose pour la prise de décision et ne fait que "brouiller les cartes" comme le soulignent Lebas et Mévellec (1999).
} 
Mais il s'agit là de la fin d'une période. A partir des années soixante-dix et pendant les années quatre-vingt les contraintes de la concurrence internationale amènent les méthodes de gestion à se moderniser.

\subsubsection{La mise en place d'un véritable contrôle de gestion}

On ne commence qu'à la fin des années soixante-dix à construire une réelle gestion budgétaire chez Usinor. Comme le fait remarquer un cadre dirigeant cité par Godelier (1995) : «On ne parlait pas tellement de coût de revient. Le premier budget que j'ai fait c'est vers 1978. Avant, c'était la comptabilité qui constatait les coûts, et le directeur qui avait l'information. De temps à autre, à peu près une fois par mois, on recevait un état de nos dépenses. On ne faisait que dépenser. A partir du moment où il y a eu la crise, on a commencé à regarder de plus près les chiffres. »

Pour analyser comment l'évolution s'est fait dans ces années charnières, nous avons pu recueillir le témoignage de deux cadres dirigeants de la sidérurgie lorraine : Mr Jupillat, qui fut membre puis responsable des équipes d'organisation et de contrôle de gestion dans les produits longs et chez Unimétal entre les années soixante et les années quatre-vingt dix (actuellement en pré-retraite) et Mr Hanicotte, qui s'occupe du contrôle et de l'analyse des coûts depuis le milieu des années soixante-dix dans la sidérurgie et qui dirige actuellement le service de contrôle de gestion de la division Sollac-Lorraine d'USINOR.

Mr Jupillat, ingénieur des Arts et Métiers, est nommé responsable du contrôle de gestion chez Sacilor au début des années soixante-dix : «On a préféré quelqu'un qui avait une bonne connaissance des procédés techniques et de l'organisation...pas un comptable, pas un financier.»(entretien avec Mr Jupillat). A cette époque: «Au niveau des responsables de service, on n'avait pas d'informations économiques : pas de prix de revient, pas de prix de vente. On ne pouvait pas s'évaluer. ». En Lorraine les fusions et restructurations successives, souvent entre des entités de taille comparable, ont suscité l'immobilisme et la neutralisation mutuelle des différentes équipes. Ce qui n'est pas favorable pour innover en matière de pratiques de gestion.

Les calculs de coûts se faisaient uniquement au niveau des patrons. Pour Mr Hanicotte : «On respectait un très grand secret en matière de production de chiffres de peur de la concurrence dans la sidérurgie. On avait affaire à des entreprises privées familiales où le comptable était l'homme de confiance du patron. C'était une question de culture patronale. »

Mr Jupillat indique que l'analyse des coûts s'est ensuite progressivement développée mais était laissée à l'instigation des chefs de services, les séances de reporting ne se tenant pas régulièrement. Dans les tableaux de bord mis en place on trouva ensuite des paramètres techniques, les prix d'achat des matières premières et les prix de revient usine. Il remarque : «On se faisait plaisir avec ces outils de contrôle de gestion. Pendant très longtemps, les enjeux stratégiques étaient autrement plus importants. Les surcoûts déterminés par les choix techniques erronés au moment des investissements étaient incomparablement plus importants que ce qu'on pouvait économiser sur place. Certaines erreurs comme la non prise en compte de la filière 
électrique à Gandrange ont ainsi généré des coûts extrêmement élevés dont on ne s'est pas remis. »

Mr Hanicotte témoigne lui aussi que l'évolution se fait très progressivement. Certes, en 1976 les pratiques se transforment dans les usines modernes de la sidérurgie sur l'eau de Dunkerque : «Aux Dunes, cela avait déjà changé. Les coûts étaient largement connus dans l'usine. Les techniciens s'appropriaient les informations sur les coûts. Ils s'y intéressaient. ». Mais, il ne s'agit encore que de coûts de revient réels. Les standards ne seront mis en place qu'au début des années quatre-vingts. Et même ainsi : «On calculait les écarts mais sans avoir de rendez-vous précis. C'était au fil de l'eau. L'animation de gestion était encore passive. ».

Il faut distinguer aussi les différents niveaux de calculs de coûts. Sur le terrain on prend des coûts d'exploitation hors amortissements. Par contre, au niveau de la direction on prend en compte les amortissements et tous les frais de structure pour se positionner de façon stratégique. On ne fait pas d'imputation rationnelle des charges fixes. Et on a eu aussi quelques pratiques très discutables. Mr Hanicotte indique à ce propos : «Le coût marginal a failli couler la sidérurgie. On justifiait de vendre au coût marginal au lieu de prendre des mesures de structure. ».

Actuellement, la Sollac-Lorraine autour du site de Florange (9 milliards de CA, 5000 personnes) gère en comptabilité 50 produits avec 140 sections et 140 vecteurs de coûts. Avec la généralisation des adaptations des produits en fonction de la demande de la clientèle, l'entreprise fabrique beaucoup plus que 50 références : «Les 50 produits comptables sont moins différenciés que ce que voit le client. On a des extras à partir des produits comptables. On fait des standards sur les références de base uniquement. On ne calcule les écarts que sur quelques produits phares ». On a là une évolution importante : «C'est nouveau cela, les extras. C'est très important pour assurer le pilotage commercial et par rapport à la marge. On a besoin d'une connaissance de plus en plus fouillée du mix-carnet. ».

On veut plus de précisions sur la relation entre rentabilité et nature des produits vendus mais on ne souhaite pas se perdre dans des calculs trop complexes au niveau des ventilations fines des coûts. Le passage à la comptabilité par activités a été discuté mais, malgré ses qualités, ce système a été jugé trop lourd. Il n'a pas été retenu. Le pilotage de gestion semble actuellement évoluer plutôt à USINOR vers la mise en place de centres de responsabilités plus autonomes et plus proches de leurs clients. C'est le sens de la récente réorganisation du groupe en divisions opérationnelles et fonctionnelles avec autonomie de gestion. On a transformé un certain nombre de centres de coûts en centres de profit. On est là très loin du mode de pilotage de la branche tel qu'il a prévalu jusqu'aux années quatre-vingt !

\section{Conclusion}

L'étude des méthodes de calcul de coûts dans la sidérurgie entre la fin de la seconde guerre mondiale (1945) et la nationalisation (1981) témoigne de l'importance de la dimension corporatiste et technocratique dans les modes de gestion de cette branche. Il y a là un exemple

\footnotetext{
${ }^{7}$ Mais chez Cockeril, concurrent belge racheté par USINOR, les amortissements étaient pris en compte déjà à ce niveau.
} 
intéressant de la façon dont on peut essayer de «construire » un environnement, et réussir en partie, mais aussi des limites d'une telle tentative face aux contraintes de l'économie réelle.

Le calcul des coûts de revient a servi dans la sidérurgie moins pour la gestion interne que pour faciliter les négociations externes avec les pouvoirs publics. C'était une démarche de type «cost plus », orientée vers la fixation des prix de vente, qui a été utilisée pendant toute la période des trente glorieuses et de l'économie administrée au niveau national.

Au cours de la crise des années soixante-dix, le modèle de régulation des patrons de la métallurgie qui faisait du calcul des coûts de revient une carte maîtresse dans les rapports de force institutionnels a été remis en cause. Une démarche de type «target costing » s'est développée, visant à satisfaire la demande des clients, dans un contexte de concurrence mondialisée. Le calcul et l'analyse des coûts est devenu alors un outil de gestion largement utilisé dans les usines et les centres de responsabilité du secteur.

Cette étude historique et sectorielle nous semble ouvrir la voie à deux types de prolongements. Bien évidemment il serait naturel, pour la compléter, de procéder à l'analyse des outils de contrôle de gestion utilisés actuellement chez USINOR pour voir leur rôle dans le pilotage organisationnel de cette grande entreprise héritière de toute la branche industrielle.

Mais l'aspect le plus intéressant serait peut-être d'approfondir la réflexion sur la nature et la place des calculs et des analyses de coûts dans des situations où la démarche reste fondamentalement de type «cost plus » en relation avec des partenaires externes. Nous pensons aux situations mettant en cause les pouvoirs publics à titre de clients ou régulateurs (comme pour les exemples déjà évoqués du marché de l'eau aux collectivités locales ou de la fixation du coût d'usage d'infrastructures de télécommunications...) mais surtout à tous les cas de partenariat étroit et de sous-traitance sur le long terme (comme dans l'automobile ou dans certains secteurs de l'électronique). Il y a là un rôle particulier de la comptabilité de gestion (dépassant une utilisation uniquement interne) qui n'est pas archaïque ou historiquement dépassé mais semble au contraire être amené à se développer de plus en plus avec la remise en cause des frontières des entreprises ${ }^{1}$.

\section{Références bibliographiques}

Archives de la C.S.S.F aux Archives nationales : fonds 62 AS 92 ; 62 AS 93.

Archives du Ministère de l'Industrie aux Archives nationales : fonds IND 22298.

Archives d'USINOR à Sérémange : fonds 10-45; 42-119; 42-18.

Bouquin H. (1993) : Comptabilité de gestion, Sirey.

Detoeuf A . (1937) : «Le problème des prix de revient» in Une méthode uniforme de calcul des prix de revient: pourquoi ? comment?, CEGOS.

Godelier E. (1995) : De la stratégie locale à la stratégie globale : la formation d'une identité de groupe chez Usinor (1948-1986), thèse, EHESS.

\footnotetext{
${ }^{8}$ Voir à ce sujet le thème annoncé pour le XXIIème congrès de l'AFC : "Entreprises sans frontières : quelles mutations pour la comptabilité et le contrôle?"
} 
Hannan et Freeman (1977) : «The population ecology of organizations », American Journal of Sociology, $\mathrm{n}^{\circ} 82$.

Kipping M. (1994) : «Les tôles avant les casseroles », Entreprises et Histoire, n5.

Lebas et Mévellec (1999) : «Vingt ans de chantiers de comptabilité de gestion », Comptabilité, Contrôle, Audit, mai. Mioche Ph. (1992) : La sidérurgie et l'Etat en France des années 40 aux années 60, Thèse, Université Paris IV.

Mioche Ph. (1993) : Jacques Ferry et la sidérurgie française, Presses de l'Université de Provence.

Nikitin M. (1992) : La naissance de la comptabilité industrielle en France, Thèse, Université Paris-Dauphine.

Nikitin M. (1996) : «Comptabilité et analyse financière à Decazeville dans les années 1830 », Entreprises et Histoire, $\mathrm{n}^{\circ} 13$.

Padioleau J. (1981) : Quand la France s'enferre, PUF.

Rimailho E. (1939) : «Contribution à l'étude d'une organisation équitable du travail par l'application d'une méthode précise d'établissement des prix de revient, le profit de l'ouvrier devenant partie intégrante du profit de l'entreprise », in Dubreuil et Rimailho, Deux hommes parlent du travail, Grasset.

Sallot J. (1993) : Le contrôle des prix et la sidérurgie française, thèse, Université Paris I.

Zimnovitch H. (1997) : Les calculs du prix de revient dans la seconde industrialisation en France, thèse, Université de Poitiers.

Zimnovitch H. (1999): «Etat et calcul des coûts par les entreprises françaises, l'exemple de Péchiney», Comptabilité, Contrôle, Audit, mars. 